\title{
Clinical features of children with chronic non-bacterial osteomyelitis: A multicenter retrospective case series from Turkey
}

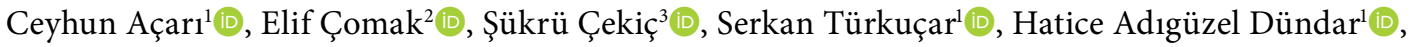

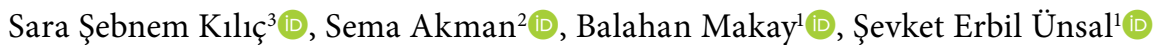 \\ ${ }^{1}$ Department of Pediatrics, Division of Pediatric Rheumatology, Dokuz Eylül University, Faculty of Medicine, Izmir, Turkey \\ ${ }^{2}$ Department of Pediatrics, Division of Pediatric Nephrology, Akdeniz University, Faculty of Medicine, Antalya, Turkey \\ ${ }^{3}$ Department of Pediatrics, Division of Pediatric Allergy-Immunology, Uludağ University, Faculty of Medicine, Bursa, Turkey
}

\begin{abstract}
Objectives: This study aims to evaluate demographic, clinical, and radiological characteristics of Turkish children with chronic non-bacterial osteomyelitis.

Patients and methods: Between January 2008 and December 2018, a total of 28 patients (10 males, 18 females; median age: 12.5 years; range, 4.5 to 21 years) who were diagnosed with chronic non-bacterial osteomyelitis in three pediatric rheumatology centers were retrospectively analyzed. The demographic, clinical, and laboratory findings of the patients were recorded.

Results: The median age at the time of diagnosis was 10.2 years. The median time from symptom onset to diagnosis was 6.5 months. The median follow-up was 18.5 months. The most frequent symptom at onset was arthralgia (75.0\%). The most frequently involved bone was the femur (67.9\%). Eight (63.6\%) of 12 patients had at least one Mediterranean fever gene (MEFV) mutation, indicating a significantly higher prevalence than the Turkish population (14.8\%). Five of these patients fulfilled the diagnostic criteria for familial Mediterranean fever (FMF). All patients received non-steroidal anti-inflammatory drugs. Other drugs were methotrexate (46.4\%), sulfasalazine (39\%), corticosteroids (25\%), anti-tumor necrosis factor (anti-TNF) agents (32\%), pamidronate (25\%), and colchicine (21.4\%). Six of eight patients with MEFV mutations were administered with colchicine, and all of them responded to treatment.

Conclusion: Clinical evolution and imaging investigations should be carefully performed to prevent any delay in the diagnosis of patients with chronic non-bacterial osteomyelitis. Based on our study results, FMF coexistence is worth investigating in patients with chronic non-bacterial osteomyelitis, particularly in the Turkish population.

Keywords: Children, familial Mediterranean fever, MEFV mutation, non-bacterial osteomyelitis.
\end{abstract}

Chronic non-bacterial osteomyelitis (CNO) is a rare inflammatory bone disease. It was first reported by Giedion et al. ${ }^{1}$ in 1972 as symmetric multifocal bone lesions. It is an orphan disease and, therefore, little is known about its diagnosis, treatment, and follow-up. The bone inflammation is defined as $\mathrm{CNO}$, regardless of the number of lesions, and chronic recurrent multifocal osteomyelitis (CRMO) is thought to be a more severe form of CNO. Nevertheless, these two terms are mostly used interchangeably. ${ }^{2}$

The cause and physiopathology of the disease are still uncertain. Although the underlying pathogenic mechanism is unknown in CNO patients, the production of immunomodulatory cytokines (interleukin [IL]-10 and IL-19) decreases,

Received: March 23, 2020 Accepted: December 28, 2020 Published online: February 08, 2021

Correspondence: Ceyhun Açarı, MD. Dokuz Eylül Üniversitesi Tıp Fakültesi Çocuk Romatolojisi Bilim Dalı, 35340 İnciraltı, İzmir, Türkiye. Tel: +90 505 - 2136359 e-mail: ceyhun_acari@hotmail.com

Citation:

Açarı C, Çomak E, Çekiç Ş, Türkuçar S, Adıguzel Dundar H, Kılıç SŞ, et al. Clinical features of children with chronic non-bacterial osteomyelitis: A multicenter retrospective case series from Turkey. Arch Rheumatol 2021;36(3):419-426.

(o2021 Turkish League Against Rheumatism. All rights reserved.

This is an open access article under the terms of the Creative Commons Attribution-NonCommercial License, which permits use, distribution and reproduction in any medium, provided the original work is properly cited and is not used for commercial purposes (http://creativecommons.org/licenses/by-nc/4.0/). 
and inflammation and proinflammatory cytokines (IL-1 $\alpha$, IL-6, and tumor necrosis factor [TNF]-alpha [TNF- $\beta$ ]) increase. ${ }^{3} \mathrm{~A}$ few genes that can cause sterile osteomyelitis (LPIN2, IL1RN, FBLIM1, PSTPIP2) have been identified in human and animal models. However, mutations in these genes have been detected in a small CNO group. ${ }^{4}$

Jansson et al. ${ }^{5}$ has proposed diagnostic criteria including clinical, laboratory, and radiological criteria; however, there is still no confirmed standard diagnostic method. Although non-steroidal anti-inflammatory drugs (NSAIDs), corticosteroids, disease-modifying anti-rheumatic drugs (DMARDs), and biological drugs are used in the treatment, there are no randomized-controlled trials for the treatment of CNO. Recently, Eurofever records have been published about demographic data and clinical, instrumental, and therapeutic features of $486 \mathrm{CNO}$ cases. $^{2}$ Accordingly, the disease can present with a range of clinical manifestations in a large patient group.

In the present study, we aimed to evaluate the clinical and laboratory characteristics of $\mathrm{CNO}$ patients from three pediatric rheumatology centers located in Turkey and to present the diagnostic methods, treatment approaches, and the place of familial Mediterranean fever (FMF) coexistence in Turkey in these specific patient populations.

\section{PATIENTS AND METHODS}

This multicenter, retrospective study included a total of 28 patients (10 males, 18 females; median age: 12.5 years; range, 4.5 to 21 years) who were diagnosed with CNO between January 2008 and December 2018 in three pediatric rheumatology centers across the country. Clinical features, laboratory data, imaging, and histopathological examination results of the patients were retrieved from medical charts and electronic files. Treatments were documented. First, infection and malignancy etiologies were ruled out in patients who had compatible lesions with CNO in at least one imaging method and/or biopsy-confirmed osteomyelitis. The diagnosis of all patients was confirmed according to the Jansson criteria (Table 1). ${ }^{5}$ A written informed consent was obtained from each parent and/or his/her legal guardian. The study protocol was approved by the Dokuz Eylül University Non-Inventional Researchs Ethics Committee (approval date/no 2019/10-17). The study was conducted in accordance with the principles of the Declaration of Helsinki.

The data recorded from patients' files included complete blood count, C-reactive protein (CRP), erythrocyte sedimentation rate (ESR), ferritin, antinuclear antibody (ANA), human leukocyte antigen (HLA)-B27, and MEFV gene mutation. An ANA (enzyme-linked immunosorbent assay [ELISA] method) of $\geq 1 / 100$ titer was accepted as positive. The HLA-B27 was analyzed in patients with sacroiliac involvement. The MEFV mutations were analyzed using the polymerase chain reaction (PCR) method.

Musculoskeletal findings were categorized as arthralgia, arthritis, bone pain, swelling, and limping. Magnetic resonance imaging (MRI), radionuclide bone scintigraphy, and

Table 1. Major and minor diagnostic criteria of chronic non-bacterial osteomyelitis according Jansson et al. ${ }^{7}$

Major diagnostic criteria

1. Radiologically proven osteolytic/-sclerotic bone lesion

2. Multifocal bone lesions

3. Palmoplantar pustulosis or psoriasis

4. Sterile bone biopsy with signs of inflammation and/or fibrosis, sclerosis
Minor diagnostic criteria
A. Normal blood count and good general state of health
B. CRP and ESR mildly-to-moderately elevated
C. Observation time longer than 6 months
D. Hyperostosis

E. Associated with other autoimmune diseases apart from palmoplantar pustulosis or psoriasis

F. Grade 1 or 2 relatives with autoimmune or autoinflammatory disease, or with NBO

CRP: C-reactive protein; ESR: Erythrocite sedimentation rate; NBO: Non-bacterial osteomyelitis; Chronic non-bacterial osteomyelitis is confirmed by two major criteria or one major and three minor criteria. 
histopathological examination findings were evaluated. Lesions were recorded according to the bone and joint localizations. Treatment regimens of all patients were also recorded. Outcomes were unable to be evaluated due to non-standardized treatment modalities among the centers.

\section{Statistical analysis}

Statistical analysis was performed using the IBM SPSS for Windows version 20.0 software (IBM Corp., Armonk, NY, USA). Descriptive data were expressed in median and interquartile range (IQR) for continuous variables and in number and percentage for categorical variables. The ShapiroWilk test was used to analyze the distribution of the data.

\section{RESULTS}

The median age at the time of diagnosis was 10.2 (IQR 5.7) years. The median diagnosis delay time was 6.5 (IQR 15.0) months, and the median follow-up was 18.5 (IQR 29.2) months (Table 2).

The onset symptoms were arthralgia $(n=21$, 75.0\%), bone pain ( $\mathrm{n}=18,64.3 \%)$, limping ( $\mathrm{n}=9,32.1 \%)$, local bone swelling $(\mathrm{n}=8,28.6 \%)$, weakness $(n=3,10.7 \%)$, and fever $(n=1,3.6 \%)$. Five patients had a history of $\mathrm{CNO}$, and two patients had a history of FMF in their first-degree relatives. Juvenile idiopathic arthritis was the most frequent preliminary diagnosis $(n=10,35.7 \%)$,

Table 2. Demographic clinics and laboratory data in pediatric patients with chronic non-bacterial osteomyelitis

\begin{tabular}{|c|c|c|c|c|}
\hline & $\mathrm{n}$ & $\%$ & Median & IQR \\
\hline \multicolumn{5}{|l|}{ Sex } \\
\hline Female & 10 & 35.7 & & \\
\hline Male & 18 & 64.3 & & \\
\hline Diagnosis age (year) & & & 10.2 & $6.1-11.8$ \\
\hline Diagnosis delay time (month) & & & 6.5 & $3.0-18.0$ \\
\hline Follow-up time (month) & & & 18.5 & $9.0-38.2$ \\
\hline \multicolumn{5}{|l|}{ Laboratory } \\
\hline Hemoglobin $(\mathrm{g} / \mathrm{dL})$ & & & 11.2 & $10.6-12.1$ \\
\hline Leukocyte $\left(10^{3} / \mathrm{mm}^{3}\right)$ & & & 8.5 & 7.4-10.2 \\
\hline Platelet $\left(10^{3} / \mathrm{mm}^{3}\right)$ & & & 433.5 & $358.2-$ \\
\hline Erythrocyte sedimentation rate $(\mathrm{mm} / \mathrm{h})$ & & & 42.0 & 563.2 \\
\hline C-reactive protein $(\mathrm{mg} / \mathrm{L})$ & & & 17.0 & $27.0-67.0$ \\
\hline Ferritin (mg/dL) & & & 42.7 & $5.0-35.2$ \\
\hline Antinuclear antibody positivity $(n=26)$ & 5 & 19.6 & & $22.4-88.8$ \\
\hline \multicolumn{5}{|l|}{ Presenting symptoms } \\
\hline Arthralgia & & 75.0 & & \\
\hline Bone pain & & 64.3 & & \\
\hline Limping & & 32.1 & & \\
\hline Local bone swelling & & 28.6 & & \\
\hline Bone biopsy & 9 & 32.1 & & \\
\hline \multicolumn{5}{|l|}{ MRI findings $(n=28)$} \\
\hline Bone marrow edema & 28 & 100.0 & & \\
\hline Hyperostosis & 4 & 14.2 & & \\
\hline Osteolytic lesion & 3 & 10.7 & & \\
\hline Increased synovial fluid & 5 & 17.8 & & \\
\hline \multicolumn{5}{|l|}{ Treatment } \\
\hline NSAIDs & 28 & 100 & & \\
\hline Sulfasalazine & 11 & 39.3 & & \\
\hline Methotrexate & 13 & 46.4 & & \\
\hline Steroids & 7 & 25.0 & & \\
\hline Colchicine & 6 & 21.4 & & \\
\hline Anti-TNF agents & 9 & 32.1 & & \\
\hline Pamidronate & 7 & 25.0 & & \\
\hline
\end{tabular}

IQR: Interquartile range; NSAIDs: non-steroidal anti-inflammatory drugs, anti-TNF: Anti-tumor necrosis factor 


\begin{tabular}{|c|c|c|c|}
\hline MEFV mutations & $\mathrm{n}=12$ & Bone involvement & Joint involvement \\
\hline M694V/V726A & 1 & Femur, pelvic bones & Sacroiliitis \\
\hline M694V/R202Q & 3 & Femur, pelvic bones, tibia & Sacroiliitis \\
\hline V726A/- & 2 & Femur, pelvic bones, tibia & No \\
\hline R202Q/- & 1 & Tibia, & Ankle \\
\hline A744S/- & 1 & Mandible & Costacondral joints \\
\hline Negative & 4 & $\begin{array}{l}\text { 1. Femur, tibia } \\
\text { 2. Femur, tibia, cranium, mandible calcaneus, } \\
\text { 3. Femur, tibia, clavicula, } \\
\text { 4. Femur, pelvic bones }\end{array}$ & $\begin{array}{l}\text { No } \\
\text { No } \\
\text { Sacroiliitis } \\
\text { No }\end{array}$ \\
\hline
\end{tabular}

followed by malignancy ( $n=9,32.1 \%)$, bacterial osteomyelitis $(n=5,17.9 \%)$, septic arthritis $(n=2$, $7.1 \%)$, Osgood-Schlatter's disease $(n=1,3.6 \%)$, and slipped capital femoral epiphysis $(n=1,3.6 \%)$.

Twenty-three (85.7\%) patients had elevated ESR (median value: $42.0 \mathrm{~mm} / \mathrm{h}$, IQR 40.0), 20 (71.4\%) patients had increased CRP (median value: $17.2 \mathrm{mg} / \mathrm{L}$, IQR 30.2), and 18 (64.2\%) patients had thrombocytosis (median value: $433.5 \times 10^{3} / \mathrm{mm}^{3}$ ). Ferritin levels were normal. HLA-B27 was positive in three of 12 (25\%) patients, and ANA was positive in five of 26 (19.6\%) patients (Table 2). Other connective tissue diseases were excluded in patients with ANA positivity. The MEFV mutations were analyzed in 12 patients. Eight had positive mutations, and seven of them were related to exon 10 . The patients had variations as M694V/V726A, M694V/R202Q, V726A/, R202Q/- and A744S/- (Table 3). Five of these patients fulfilled the diagnostic criteria (Yalcinkaya-Ozen criteria) for FMF. ${ }^{6}$ Familial cases (two siblings) were investigated for MEFV, IL1RN, PSTPIP2, and LPIN2 mutations, which were found to be negative.

All patients had X-ray and MRI imaging. Whole-body MRI was not available. On MRI, bone marrow edema was determined in all 28 patients, while other findings were related to hyperostosis (14.2\%), osteolytic lesions (10.7\%), and increased synovial fluid in adjacent joints (17.8\%). There was an increased osteoblastic activity in all 14 patients who had radionuclide bone scintigraphy.
Twenty-seven patients, except for one, had multifocal involvement. The most affected bones were femur in 19 (67.9\%) patients, followed by tibia in 16 (57.1\%) patients, pelvic bones in nine (32.1\%) patients, tarsal bones in six (21.4\%) patients, and clavicle in four (14.3\%) patients. Others were mandible $(n=3)$, vertebra $(n=2)$, metatarsi $(n=2)$, sacrum $(n=1)$, and cranium $(n=1)$ (Figure 1). There was a prominent metaphyseal involvement (60.7\%). Other localizations were metaphysoepiphysial (21.4\%), metaphysodiaphysial (14.3\%), and diaphyseal (3.6\%). In patients with MEFV mutations, the mainly affected regions were pelvic bones, tibia, and femur metaphysis, respectively (Table 3).

Nine patients had a bone biopsy. Histopathological examination of six patients revealed a cell infiltration which was a mixture of lymphocytes and neutrophil cells. Neutrophil infiltration was seen in two patients, and one patient had only lymphocyte infiltration. Microbial analyses were negative in all patients.

Two cases had keratosis pilaris and xeroderma pigmentosum. However, there was no $\mathrm{CNO}$ associated with skin lesions, such as acne, palmoplantar pustulosis, or psoriasis. Two patients had inflammatory bowel disease (colitis ulcerosa, Crohn's disease). Eight patients had MEFV mutations, and five of them were diagnosed as FMF. Three patients met the International League Against Rheumatism- Enthesitis related arthritis/Juvenile spondyloarthropathy (ILAR ERA/JSpA) criteria. ${ }^{[7]}$ 


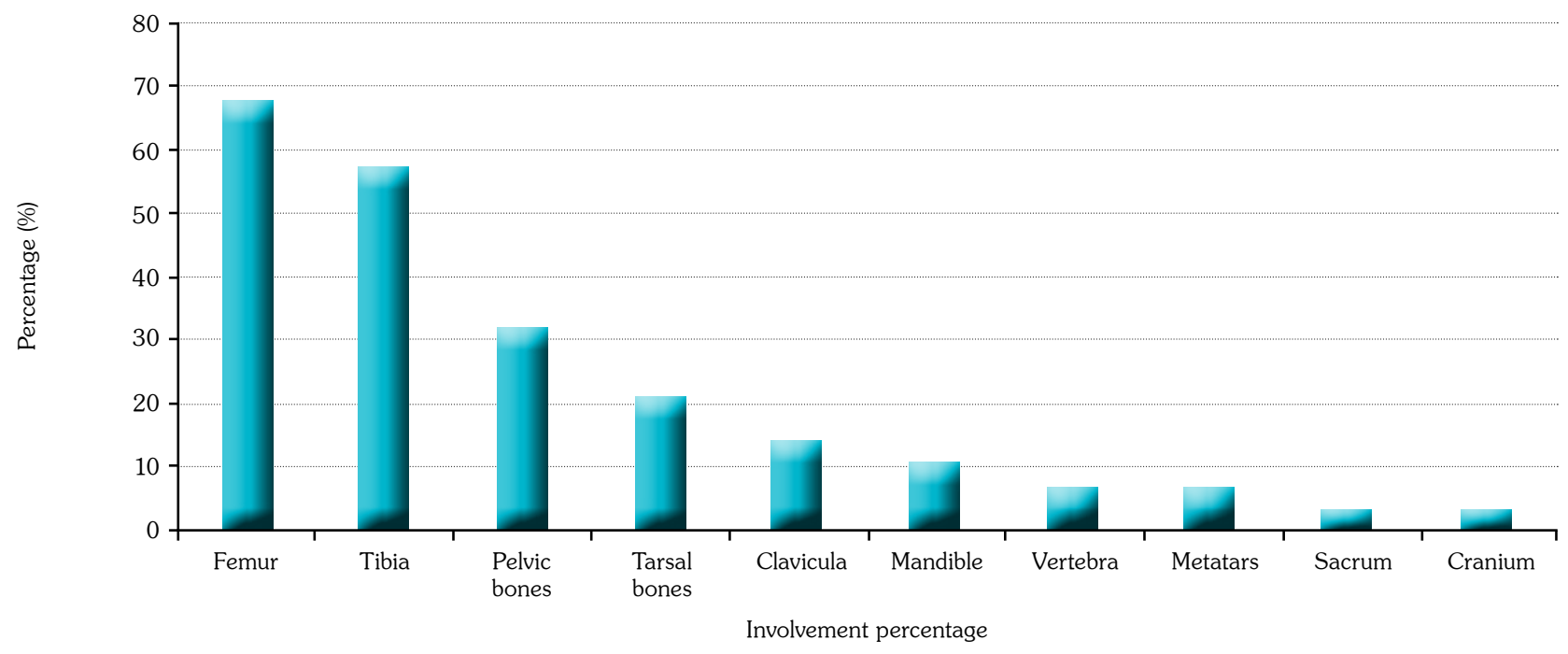

Figure 1. Bone involvement in 28 pediatric patients with chronic non-bacterial osteomyelitis.

All the patients used NSAIDs. The DMARD treatment included methotrexate (46.4\%), sulfasalazine (39.3\%), corticosteroids (25\%), and colchicine for the ones with MEFV mutation (21.4\%). Anti-TNF drugs were administered to nine patients unresponsive to DMARDs (32.1\%), while four of them were additionally administered with pamidronate (25\%) to control the disease. Six patients with MEFV mutations received sulfasalazine $(n=6)$, corticosteroids $(n=3)$, antiTNF $(\mathrm{n}=3)$, pamidronate $(\mathrm{n}=2)$, and methotrexate $(\mathrm{n}=1)$, in addition to colchicine. The patients who poorly responded to previous treatments achieved improvement after the initiation of colchicine.

\section{DISCUSSION}

In the present study, we evaluated the clinical and laboratory features, radiological findings, and treatments of $28 \mathrm{CNO}$ Turkish patients followed in three different pediatric rheumatology centers. Our study results showed that FMF coexistence is worth investigating in patients with chronic non-bacterial osteomyelitis, particularly in the Turkish population.

The Eurofever study evaluated $486 \mathrm{CNO}$ patients, 455 of whom were pediatric patients. ${ }^{2}$ Accordingly, there was a female predominance (2:1). The age of onset of the disease was 9.9 (range, 1 to 17.7) years, and the age of diagnosis was 10.9 (range, 1.4 to 17.7 ) years. Symptoms at the time of admission were bone pain (92\%), arthralgia (65\%), bone deformity (15\%), and skin manifestation (i.e., acne, psoriasis, palmoplantar pustulosis) (19\%). Sağ et al. $^{8}$ reported the female/male ratio as $7 / 8$, disease onset age as 9 (0.6-15), diagnosis age as 10.5 (3-16.5), and diagnosis delay time as 23 months. Symptoms at the time of admission were bone pain (93.4\%) and limping (6\%), and only one patient had cutaneous findings (skin abscess). There was no female dominance in the present series (F/M; 10/18). This finding was similar to the Latin American and Asian series. ${ }^{9}, 10$ While the age of diagnosis and the age of onset were similar, the diagnostic delay time was shorter (6.5 months). The onset symptoms were mainly arthralgia, bone pain, and limping (75.3\%, 64.3\%, and 32.1\%, respectively). Skin lesions associated with $\mathrm{CNO}$ were not observed in any patients, which is similar to the Asian and Latino series. ${ }^{9,10}$

According to Eurofever registry, lower extremity involvement was common and vertebral involvement was present in $23 \%$ of patients. ${ }^{2}$ Sağ et $a .^{8}$ reported the vertebral involvement rate as $66.6 \%$. They suggested the use of more aggressive treatment in patients with vertebral lesions to prevent complications. ${ }^{8}$ Vertebrae, clavicle, mandible, and upper extremities were less involved than the European and North American patients. ${ }^{11-13}$ In the present study, 
lesions were most common in femur and tibia, while vertebral involvement was observed in only two patients (7.1\%).

Sözeri et al. ${ }^{14}$ reported 17 patients with $\mathrm{CNO}$ in their study, which included the second-largest cohort from Turkey after the present study. Their retrospective study included the demographic data and clinical features of 17 children diagnosed with CRMO in two pediatric rheumatology centers. Their report indicated that CRMO might be overlooked and misdiagnosed at first sight in our country, suggesting a closer follow-up of these patients to avoid potential complications and sequelae.

Batu et al. ${ }^{15}$ reported that four of five $\mathrm{CNO}$ patients had sacroiliitis. They suggested that there was a relationship between $\mathrm{CNO}$ and juvenile spondyloarthritis (JSpA). Skrabl-Baumgartner et al. ${ }^{16}$ reported that one-third of pediatric $\mathrm{CNO}$ patients fulfilled the International League of Associations of Rheumatology (ILAR) enthesitisrelated arthritis (ERA)/JSpA criteria. Vittecoq et al. ${ }^{17}$ also showed that most of the $\mathrm{CNO}$ patients evolved into JSpA over a 10 -year period. This similarity raised the question of whether $\mathrm{CNO}$ was an initial form of JSpA. In our study, HLA-B27 was positive in three of 12 patients with sacroiliac involvement and these three patients met the ILAR ERA/JspA criteria.

In the study of Silier et al., ${ }^{11}$ preliminary diagnoses were malignancy (36\%), bacterial osteomyelitis (30\%), arthritis (15\%), fracture (15\%), and growth pain (4\%). In the present study, the lesions were mostly multifocal and localized in metaphysis, which is similar to the study by Silier et al. ${ }^{11}$ Frequent preliminary diagnoses of patients were juvenile idiopathic arthritis, bacterial osteomyelitis, and septic arthritis in the present study. The most involved bones were the femur, tibia, and pelvis.

In another study, Girschick et al. ${ }^{13}$ reported that laboratory tests were non-specific for diagnosis, while a mild increase in acute phase reactants accompanied an exacerbation or might be related to multiple lesions. The present study had similar results with the Eurofever study in terms of acute phase response. $^{2}$

Magnetic resonance imaging is the preferred imaging modality to characterize lesions at onset and to monitor treatment response. It has an important value for diagnosis, as well as medical history and physical examination. Whole-body MRI is very helpful in applicable centers. ${ }^{18}$ The radionuclide bone scintigraphy detected increases in the osteoblastic activity. In the present study, primary MRI findings revealed bone marrow edema, effusion in joints, hyperostosis, and osteolytic lesions. Imaging findings of $\mathrm{CNO}$ patients were compatible with the Eurofever registry. ${ }^{2}$ In the light of the current literature and our study results, imaging modalities can show the non-specific inflammation in the tissue; however, they cannot indicate any pathognomonic finding.

The histopathological evaluation may be required in some cases for the differential diagnosis. In particular, it is preferred in cases in whom malignancy must be ruled out. ${ }^{19}$ In the present study, a histopathological examination was performed in nine (32.1\%) patients. There was a sterile non-specific composition of lymphocytes and neutrophils in the specimens.

The relevant literature contains case series of $\mathrm{CNO}$ patients having MEFV mutations who received additional benefits from colchicine treatment. All of these patients had exon 10 mutations. ${ }^{20-23}$ Colchicine treatment was started in a girl with osteomyelitis and febrile attacks who had MEFV mutations (E148Q/ P369S/R408Q). ${ }^{20}$ The lower extremity osseous lesions improved after colchicine for one year; however, she had another episode of osteomyelitis, when she stopped taking colchicine. Two CNO cases from Turkey were reported with compound heterozygote MEFV (exon 10) mutations (M694V/E148Q and M694V/M680I) and received benefits from colchicine. ${ }^{21}$ Moussa et al. ${ }^{22}$ described seven cases with $\mathrm{CNO}$, with one of them having FMF with heterozygote M694V mutation. The patient had vertebral, pelvic, and lower extremities involvement. Naproxen, methotrexate, and pamidronate were administered respectively, along with colchicine. A recent study reported a CNO patient with FMF (V726A heterozygote), who responded well to anakinra. ${ }^{23}$ She had the involvement of femoral condyles and proximal tibia. There are no studies investigating the relationship between $\mathrm{CNO}$ and FMF, except for a few number of case reports and case series. The Eurofever registry, the largest $\mathrm{CNO}$ cohort, did not show a possible 
relationship between $\mathrm{CNO}$ and $\mathrm{FMF}^{2}$ It may be speculated that MEFV mutations, mainly exon 10, may have an inflammatory stimulation of bones with $\mathrm{CNO}$, and additional colchicine treatment is helpful in the disease control. Sözeri et al. ${ }^{14}$ detected MEFV gene mutations in four patients in whom severity of clinical features reduced with colchicine therapy. Soylemezoglu et al. ${ }^{24}$ also found a much higher FMF prevalence (14.8\%) than expected in the Turkish population (14.8\%). In the present study, at least one MEFV mutation was detected in eight of 12 (63.6\%) patients who were assessed for FMF coexistence. Seven of these patients (88\%) had an exon 10 mutation, and five met the diagnostic criteria for FMF and were administered with colchicine. The MEFV positive group mainly had involvement of pelvic bones and femur. Furthermore, 50\% with M694V allele had sacroiliitis. This can be evaluated as the most interesting and striking result of the present study. Thus, searching MEFV mutations can be recommended in patients living in countries where FMF is common, particularly when the lesions are resistant to common treatment modalities.

The retrospective design with a relatively small sample size can be deemed as limitations of the present study. In addition, the clinical outcomes of patients were unable to be assessed, due to the lack of standardized treatment modalities among the study centers. Nonetheless, being the largest cohort of CNO patients from Turkey is the main strength of this study.

In conclusion, $\mathrm{CNO}$ is a chronic inflammatory disease of bone that may have a progressive or relapsing course. The disease still has many aspects to be discovered. In addition, FMF is the main accompanying disease to $\mathrm{CNO}$ in the Mediterranean region, which has a role in clinical manifestation and response to treatment. Considering the results of this study, it can be speculated that FMF coexistence is always worth investigating in these specific patient populations, particularly in countries such as Turkey which have a high FMF patient prevalence.

\section{Declaration of conflicting interests}

The authors declared no conflicts of interest with respect to the authorship and/or publication of this article.

\section{Funding}

The authors received no financial support for the research and/or authorship of this article.

\section{REFERENCES}

1. Giedion A, Holthusen W, Masel LF, Vischer D. Subacute and chronic "symmetrical" osteomyelitis. Ann Radiol (Paris) 1972;15:329-42.

2. Girschick H, Finetti M, Orlando F, Schalm S, Insalaco A, Ganser G, et al. The multifaceted presentation of chronic recurrent multifocal osteomyelitis: a series of 486 cases from the Eurofever international registry. Rheumatology (Oxford) 2018;57:1203-11.

3. Schnabel A, Range U, Hahn G, Berner R, Hedrich $\mathrm{CM}$. Treatment response and longterm outcomes in children with chronic nonbacterial osteomyelitis. J Rheumatol 2017;44:1058-65.

4. Cox AJ, Ferguson PJ. Update on the genetics of nonbacterial osteomyelitis in humans. Curr Opin Rheumatol 2018;30:521-5.

5. Jansson A, Renner ED, Ramser J, Mayer A, Haban $M$, Meindl $A$, et al. Classification of non-bacterial osteitis: retrospective study of clinical, immunological and genetic aspects in 89 patients. Rheumatology (Oxford) 2007;46:154-60.

6. Yalçinkaya F, Ozen S, Ozçakar ZB, Aktay N, Cakar $\mathrm{N}$, Düzova $\mathrm{A}$, et al. A new set of criteria for the diagnosis of familial Mediterranean fever in childhood. Rheumatology (Oxford) 2009;48:395-8.

7. Colbert RA. Classification of juvenile spondyloarthritis: Enthesitis-related arthritis and beyond. Nat Rev Rheumatol 2010;6:477-85.

8. Sağ E, Sönmez HE, Demir S, Bilginer Y, Ergen FB, Aydıngöz Ü, et al. Chronic recurrent multifocal osteomyelitis in children: a single center experience over five years. Turk J Pediatr 2019;61:386-91.

9. Concha S, Hernández-Ojeda A, Contreras O, Mendez C, Talesnik E, Borzutzky A. Chronic nonbacterial osteomyelitis in children: a multicenter case series. Rheumatol Int 2020;40:115-20.

10. Rao AP, Mallya PP, Ranjani S, Raghuram J. Chronic recurrent multifocal osteomyelitis - a case series from India. Indian J Orthop 2018;52:672-7.

11. Silier CCG, Greschik J, Gesell S, Grote V, Jansson AF. Chronic non-bacterial osteitis from the patient perspective: a health services research through data collected from patient conferences. BMJ Open 2017;7:e017599.

12. Borzutzky A, Stern S, Reiff A, Zurakowski D, Steinberg EA, Dedeoglu F, et al. Pediatric chronic nonbacterial osteomyelitis. Pediatrics 2012;130:e1190-7.

13. Girschick HJ, Raab P, Surbaum S, Trusen A, Kirschner S, Schneider $P$, et al. Chronic nonbacterial osteomyelitis in children. Ann Rheum Dis 2005;64:279-85. 
14. Sözeri B, Ayaz NA, Atıkan BY, Karadağ ŞG, Çakan $\mathrm{M}$, Argin $\mathrm{M}$, et al. Clinical experiences in Turkish paediatric patients with chronic recurrent multifocal osteomyelitis. Turk J Pediatr 2019;61:879-84.

15. Batu ED, Ergen FB, Gulhan B, Topaloglu R, Aydingoz $\mathrm{U}$, Ozen $\mathrm{S}$. Etanercept treatment in five cases of refractory chronic recurrent multifocal osteomyelitis (CRMO). Joint Bone Spine 2015;82:471-3.

16. Skrabl-Baumgartner A, Singer $P$, Greimel $T$, Gorkiewicz G, Hermann J. Chronic non-bacterial osteomyelitis: a comparative study between children and adults. Pediatr Rheumatol Online J 2019;17:49.

17. Vittecoq O, Said LA, Michot C, Mejjad O, Thomine $\mathrm{JM}$, Mitrofanoff $\mathrm{P}$, et al. Evolution of chronic recurrent multifocal osteitis toward spondylarthropathy over the long term. Arthritis Rheum 2000;43:109-19.

18. Depasquale R, Kumar N, Lalam RK, Tins BJ, Tyrrell PN, Singh J, et al. SAPHO: What radiologists should know. Clin Radiol 2012;67:195-206.

19. Kaiser D, Bolt I, Hofer M, Relly C, Berthet G, Bolz D, et al. Chronic nonbacterial osteomyelitis in children: a retrospective multicenter study. Pediatr Rheumatol Online J 2015;13:25.
20. Shimizu M, Tone Y, Toga A, Yokoyama T, Wada T, Toma T, et al. Colchicine-responsive chronic recurrent multifocal osteomyelitis with MEFV mutations: a variant of familial Mediterranean fever? Rheumatology (Oxford) 2010;49:2221-3.

21. Gezgin Yildirim D, Bedir Demirağ T, Akdulum İ, Yılmaz G, Bakkaloğlu S. Chronic non-bacterial osteomyelitis coexistent with familial Mediterranean fever. Clin Exp Rheumatol 2018;36(6 Suppl 115): 150 .

22. Moussa T, Bhat V, Kini V, Fathalla BM. Clinical and genetic association, radiological findings and response to biological therapy in seven children from Qatar with non-bacterial osteomyelitis. Int $\mathrm{J}$ Rheum Dis 2017;20:1286-96.

23. Babaoglu H, Varan O, Kucuk H, Turan A, Tufan A. Osteitis as a manifestation of familial Mediterranean fever. Rheumatology (Oxford) 2017;56:2035-6.

24. Soylemezoglu O, Kandur Y, Gonen S, Düzova A, Özçakar ZB, Fidan K, et al. Familial Mediterranean fever gene mutation frequencies in a sample Turkish population. Clin Exp Rheumatol 2016;34(6 Suppl 102):97-100. 